\title{
Compact imaging Bragg spectrometer for fusion devices
}

\author{
G. Bertschinger, ${ }^{\text {a) }}$ W. Biel, H. Jaegers, and O. Marchuk \\ Institute of Plasma Physics, Forschungszentrum Jülich GmbH, EURATOM Association, Trilateral Euregio \\ Cluster, D-52425 Jülich, Germany
}

(Presented on 20 April 2004; published 6 October 2004)

\begin{abstract}
A compact imaging $\mathrm{x}$-ray spectrometer has been designed for tokamaks and stellarators to measure the plasma parameters at different spatial chords. It has been optimized for high spectral resolution and high sensitivity. High spectral resolution is obtained by using solid state detectors and minimizing the imaging errors of the spherical crystals. It is shown, that using spherical crystals the solid angle and hence the throughput can be increased significantly, without compromising the spectral resolution. The design is useful for the measurement of the spectra of He- and H-like ions from $\mathrm{Si}$ to $\mathrm{Kr}$. The spectral resolution is sufficient for the measurement of plasma parameters. The temporal resolution is high enough for transport studies by gas puff and laser ablation experiments. The design is based on a modified Johann spectrometer mount, utilizing a spherically bent crystal instead of the cylindrically bent crystal in the traditional Johann mount. The astigmatism of the wavelength selective reflection on the spherical crystal is applied to obtain imaging of an extended plasma source on a two-dimensional detector. For each element, a separate crystal is required, only in few cases, a crystal can be used for the spectra of two elements. For the spectra of most of the He-like ions from $\mathrm{Si}$ up to $\mathrm{Kr}$, suitable crystal cuts have been found on quartz, silicon and germanium crystals with Bragg angles in a small interval around the design value of $53.5^{\circ}$. All of the crystals have the same radius. They are fixed on a rotational table. The distance to the detector is adjusted by an $x-y$ table to fit to the Rowland circle. () 2004 American Institute of Physics. [DOI: 10.1063/1.1781755]
\end{abstract}

\section{INTRODUCTION}

$\mathrm{X}$-ray spectroscopy is a powerful method to determine basic plasma parameters of hot thermonuclear plasmas. Electron and ion temperatures as well as the plasma composition can be measured with high precision. Up to now, the x-ray spectrometers on the large experiments for magnetic confined plasmas have been Bragg spectrometers in Johann mount. These instruments use cylindrically bent crystals to obtain one-dimensional spectra on a linear detector. They have been single chord devices, which observe the plasma on a single line of sight.

Recently, it has been shown that the traditional designs can be upgraded to get simultaneously spatially resolved spectra over multiple chords. ${ }^{1}$ Both, two dimensionally bent crystals, as well as two-dimensional detectors must be used. The guidelines for such a layout have been discussed in more detail in Ref. 2.

\section{DESCRIPTION OF THE INSTRUMENT}

For TEXTOR, a medium sized tokamak, and for the large stellarator experiment W7-X, a design has been developed to measure the spectra of the He-like impurities with intermediate nuclear charge between silicon and krypton. For TEXTOR, the range of the elements will extend up to copper, as the plasma temperature is not high enough to ionize krypton up to the He-like state.

The design of the instrument on TEXTOR fulfills the following guidelines:

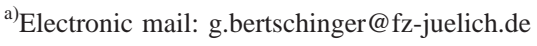

(i) The instrument is rather compact as the space around the TEXTOR experiment is limited.

(ii) The spectral resolution must be high enough to resolve the Doppler widths of the spectral lines.

(iii) The sensitivity must be as high as possible, and hence the useful area of the crystal must be as large as practical.

(iv) The time resolution of the instrument must be sufficient to follow transport studies of the impurities, therefore a time resolution of about $1 \mathrm{~ms}$ is necessary.

(v) The setting to the different elements must be done by remote control within a few seconds, for TEXTOR, it will be done between the discharges. For the long pulses on W7-X it must be possible to switch within seconds to the desired element during the discharge.

(vi) The adjustment of the instrument must be simple and performed in visible light, this excludes mounts of the spectrometer with asymmetric cuts of the crystal.

The design is based on two-dimensionally bent Bragg crystals with the crystal planes parallel to the surface. For simplicity, spherically bent crystals are chosen. In principle, toroidally bent crystals would grant more flexibility in the design. But it will be shown, the spherically bent crystals allow larger crystal areas and therefore higher sensitivity and are easier to align.

\section{FOCUSING PROPERTIES OF SPHERICALLY BENT CRYSTALS}

A crystal behaves as a wavelength selective mirror due to Bragg reflection: 


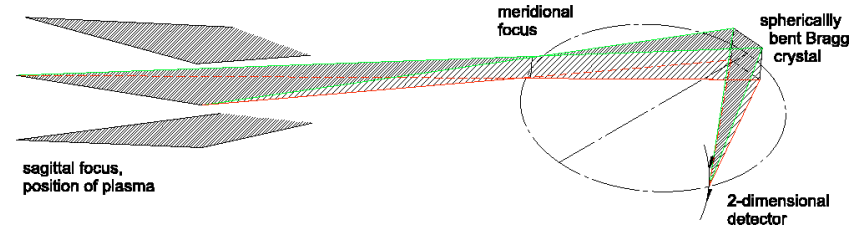

FIG. 1. (Color online) Focusing properties of a spherically bent Bragg crystal, similar to a spherical mirror with off-axis illumination.

$$
n \cdot \lambda=2 \cdot d \sin (\alpha)
$$

with $n$ : diffraction order, $\lambda$ : wavelength of the $\mathrm{x}$ rays, $\mathrm{d}$ : spacing between the crystal planes, $\alpha$ : the diffraction angle relative to the surface.

A spherically bent Bragg crystal suffers from the same astigmatism as a spherical mirror, when it is illuminated off axis. Instead of a single focus, there are two foci, a meridional focus and a sagittal focus with focal lengths:

$$
f m=\frac{R}{2} \sin (\alpha) \text { and } f s=\frac{R}{2} \cdot \frac{1}{\sin (\alpha)},
$$

respectively, where $\mathrm{R}$ is the radius of the crystal. With the lens equation:

$$
\frac{1}{f}=\frac{1}{a}+\frac{1}{b}
$$

and the distance from the crystal to the detector on the Rowland circle: $a=R \sin (\alpha)$ we arrive at the distances to the meridional and the sagittal focus: $b m=R \sin (\alpha)$, and $b s=$ $-R \cdot[\sin (\alpha)] / \cos (2 \cdot \alpha)$, respectively.

The meridional focus coincides with the focusing condition for a traditional Johann spectrometer. An image point on the Rowland circle is focused to two slit-like foci (Fig. 1). If the plasma is placed at the position of the sagittal focus and a slit at the meridional focus, a monochromatic onedimensional image of the plasma will show up on a detector. Without a slit, a spectrum will show up in the dispersion plane and an image of the plasma in the perpendicular dimension. Of course, the plasma parameters must not vary along the slit-like sagittal focus, therefore the sagittal focus must be parallel to the magnetic axis of the tokamak or the

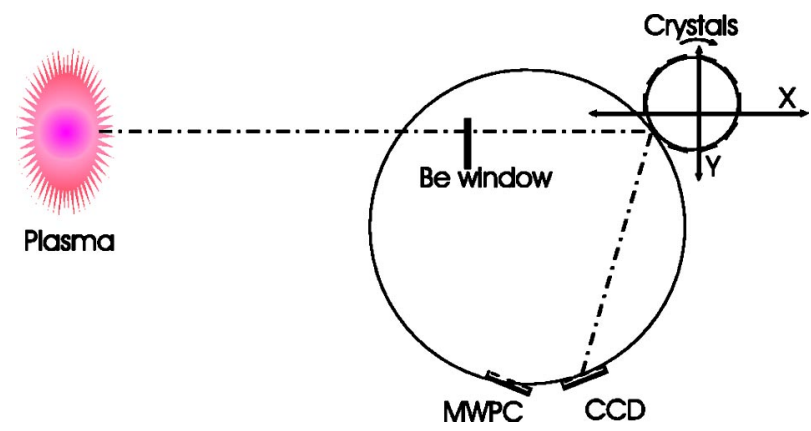

FIG. 2. (Color online) Principle of the imaging Bragg spectrometer for the spectra of He- and H-like ions. The crystals are located on a carousel and are selected via remote control. Focusing is fine tuned by adjusting the $x-y$ table. Two detectors will be used: a 2D multi-wire-proportional-counter (MWPC) with delay line readout and six open CCD detectors. The MWPC is perpendicular to the line of sight, the CCD tangential to the Rowland circle.

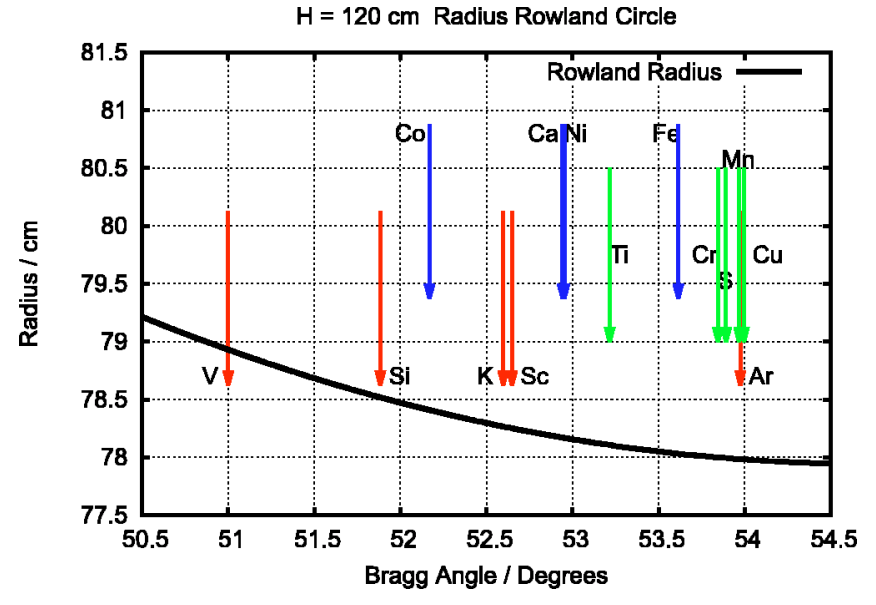

FIG. 3. (Color online) Rowland circle radius for a distance of $120 \mathrm{~cm}$ between the detector and the line of sight at Bragg angles around $54^{\circ}$. Arrows indicate crystal cuts (upper (blue) germanium, middle (green) silicon, and lower (red) quartz) for the He-like resonance line $w$ of the most important elements between $\mathrm{Si}$ and Ni (Ref. 2).

stellarator, respectively. The Bragg reflection is rotational symmetric to the normal of the Bragg crystal, therefore the focal lines on the detectors are curved.

The ratio between the saggital and the meridional focus and hence the demagnification of the plasma on the detector is then

$$
\gamma=\frac{b s}{b m}=\frac{-1}{\cos (2 \cdot \alpha)} .
$$

A real image shows up for Bragg angles $\alpha>45 \mathrm{deg}$, for $\alpha=45 \mathrm{deg}$, the focus is infinite and for $\alpha<45 \mathrm{deg}$, there is no focus. For Bragg angles close to $90 \mathrm{deg}$, the two foci coincide. For TEXTOR, the space requires a ratio $\gamma$ of about 3.5 , the Bragg angle is therefore around 54 degrees.

For the He-like spectra of the ions between $\mathrm{Si}$ and $\mathrm{Kr}$, cuts of quartz, silicon, or germanium crystals can be found in the range between 49 and $57 \mathrm{deg}$, most of the crystals cover the range between 51 and $55 \mathrm{deg} .{ }^{2}$ Now the search for suitable crystal cuts is simplified greatly by the excellent web tool "xoh-search". 3

Due to the small variation of the Bragg angle, a straightforward design is applied (Fig. 2). The position of the detector arrays is fixed and the crystals are mounted on a carousel, which can be adjusted by linear $x-y$ drives (Fig. 2). It turns out, that the variation in the $y$-coordinate is rather small (Fig. 3). In Fig. 4, the distance from the crystal to the plasma is compared with the sagittal image length. Around $53.5 \mathrm{deg}$ they agree, outside the design angle they differ and the spatial resolution is reduced slightly.

\section{X-RAY DETECTORS}

For low energy $x$ rays $(E<10 \mathrm{keV})$, two types of detectors have overall sensitivities above $50 \%$ : gas detectors such as multi-wire-proportional counters (MWPC) or solid state detectors with direct conversion of the $\mathrm{x}$ rays. Both types have been used on fusion devices and have specific properties. The gas detectors are sensitive to single $\mathrm{x}$ ray photons and have been in use over several decades, suitable solid 


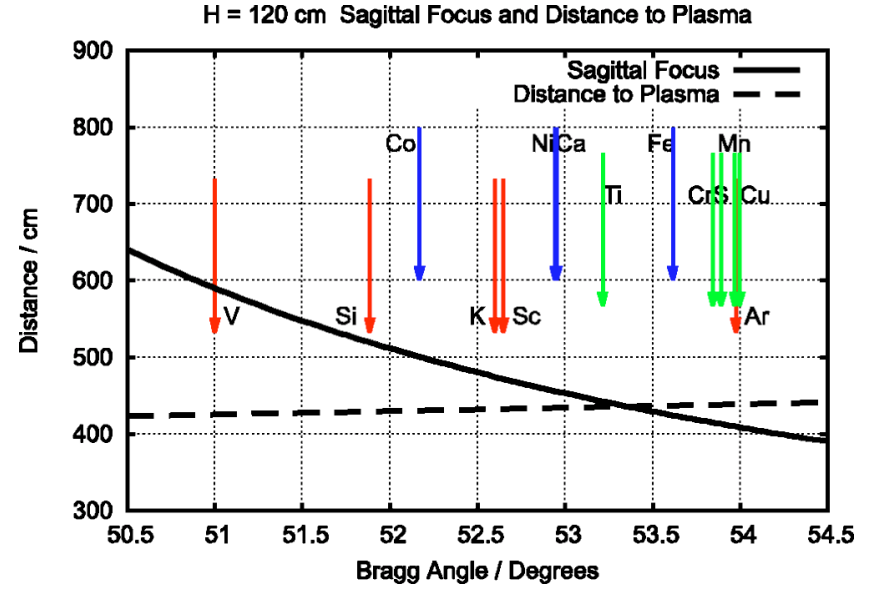

FIG. 4. (Color online) Distance from the crystal to the sagittal focus (solid) and to the plasma (dashed) for the same crystals as in Fig. 3. For 53.5 the sagittal focus is in the plasma center.

state detectors based on CCD have been developed for the recent $x$ ray satellite missions. Gas detectors have proven to be robust against hard $\mathrm{x}$ rays and neutron radiation, solid state detectors suffer from permanent damage and must be replaced at regular intervals. Whereas the gas detectors can be built with large areas, the sensitive area of the solid state detectors is rather small and limited to several $\mathrm{cm}^{2}$.

For TEXTOR, both types of detectors are foreseen: an array of six deep depletion CCD detectors with dimensions of $26.6 \mathrm{~mm}$ wide $\times 6.6 \mathrm{~mm}$ high and multiwire proportional counters with delay line readout. The CCD are chosen for their good spatial resolution, high sensitivity and high dynamic range. They are used as linear detectors by binning in the vertical dimension for fast readout of $\approx 3 \mathrm{~ms}$ per spectrum. For faster events, a set of three two-dimensional MWPC will be used. The CCD detectors will provide high resolution spectra with precise wavelengths, whereas the MWPC can follow fast events with medium wavelength resolution.

\section{WAVELENGTH RESOLUTION OF SPHERICAL CRYSTALS}

Besides the low order errors such as the astigmatism, which is used for spatial imaging, the crystal has higher order errors, which reduce the wavelength resolution and provide an upper limit for the size of the crystal.

Much effort has been dedicated to maximize the resolution of the crystals. The imaging quality is of minor importance, as the plasma parameters vary slowly in the hot core of a thermonuclear plasma and imaging errors of a few $\mathrm{cm}$ in the plasma can be tolerated. A ray-tracing program has been developed, to study the geometrical errors of the imaging with spherical, cylindrical, and toroidal crystals. With a spherical crystal, the dominant error is the Johann error, a second order error which is due to the finite length of the crystal. The finite height of the crystal is of minor importance only, as this error vanishes in second order for spherical crystals. But for cylindrical and toroidal crystals, it is of the same order as the Johann error.

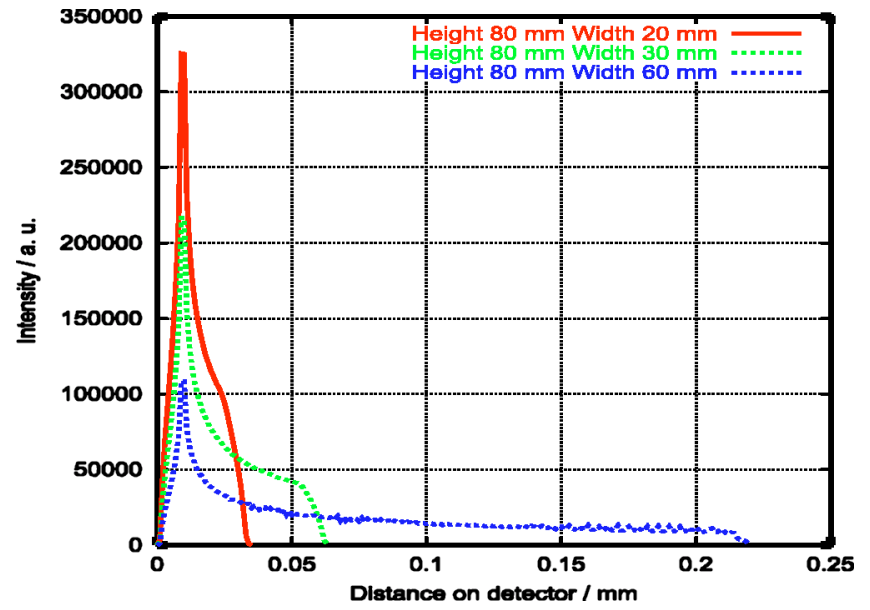

FIG. 5. (Color online) Resolution of a spherical crystal calculated for $x$ rays hitting a detector tangential to the Rowland circle for different crystal sizes. The most important error (Johann error) is second order and increases with the square of the dimension of the crystal in the dispersion plane. The image is asymmetric. For medium sized crystals, the error exceeds the resolution of the detector and the rocking curve (Rowland radius $0.8 \mathrm{~m}$, Bragg angle $54^{\circ}$ ).

\section{RESULTS OF THE RAY TRACING CALCULATIONS, GEOMETRICAL ERRORS}

The resolution is calculated for an extended source, assuming that the crystal is illuminated uniformly. The crystal is rectangular, the Bragg angle is $54 \mathrm{deg}$. The finite resolution of the crystal due to the rocking curve is not taken into account.

The distribution of the intensity on the detector is shown in Fig. 5 for different crystal sizes. For a solid state detector, the size of the crystal in the dispersion plane must be restricted to $30 \mathrm{~mm}$. For a gas counter with a resolution of $0.2 \mathrm{~mm}$, the size can extend up to $60 \mathrm{~mm}$. The height of the crystal is not restricted by the geometrical errors. As the Bragg reflection is axially symmetric, the finite width of the detector does not matter, as long as a two-dimensional detector is used. Similar results have been found by analytic expansion. ${ }^{4}$ In principle, larger crystals could be applied, by shaping the surface of the crystal similar to the Johannson mount of x-ray spectrometers with cylindrical crystals. The radii of the crystal planes, as well as the radius in vertical direction are the same as with the cylindrical crystals, whereas the surface in the dispersion plane is shaped to half radius. The resolution is improved significantly, but the production of the crystal is much more complicated. ${ }^{2}$

Compared to the traditional Johann spectrometers, and even the Johannson spectrometers, the sensitivity of spectrometers with spherical crystals is much higher, as the height of the crystals is limited only by the size of high quality crystals. Additionally, they provide information on the spatial distribution of the plasma parameters and the plasma composition.

\footnotetext{
${ }^{1}$ M. Bitter et al., Rev. Sci. Instrum. 70, 292 (1999).

${ }^{2}$ G. Bertschinger, M. Bitter, and D. Rusbüldt, in Advanced Diagnostics for Magnetic and Inertial Fusion (Kluwer Academic / Plenum, New York, 2002), p. 269.

${ }^{3}$ S. Stepanov, http://sergey.gmca.aps.anl.gov/

${ }^{4}$ J. Eggs and K. Ulmer, Z. Angew. Phys. 20, 118 (1965).
} 